\title{
Monthly rainfall forecasting by a hybrid neural network of discrete wavelet transform and deep learning
}

\author{
Xueyi You ${ }^{1}$ and Ming Wei ${ }^{1}$ \\ ${ }^{1}$ Tianjin University
}

December 14, 2021

\begin{abstract}
Actual rainfall forecast is critical to the management and allocation of water resources. In recent years, deep learning has been proved to be superior to traditional forecasting methods when predicting rainfall time series with high temporal and spatial variability. In this study, the discrete wavelet transform (DWT) and two typical deep learning approaches, namely long-short term memory (LSTM) and dilated causal convolutional neural network (DCCNN), are integrated innovatively and the hybrid model (DWT-CLSTM-DCCNN) is used for monthly rainfall forecasting for the first time. Monthly rainfall time series of four major cities in China (Beijing, Tianjin, Chongqing and Guangzhou) are used as the dataset of DWT-CLSTM-DCCNN. Firstly, two methods of sample construction are used to train DWT-CLSTM-DCCNN and their effects on the model performance are analyzed. Then, LSTM and the dilated causal convolutional network (DCCNN) are established as the benchmark models, and their forecast accuracy is compared with that of DWT-CLSTM-DCCNN. From the results of the evaluation criteria such as mean absolute error (MAE), root mean squared error (RMSE) and Nash-Sutcliffe model efficiency coefficient (NSE) as well as the fitting curve for forecasted rainfall, it can be concluded that the DWT-CLSTM-DCCNN developed in this study outperforms the benchmark models in model accuracy, peak and mutational rainfall capturing ability. Compared with the previous studies, DWT-CLSTM-DCCNN is proven to be better peak capture and more suitable for long-term rainfall forecasting.
\end{abstract}

\section{Hosted file}

Main Document.docx available at https://authorea.com/users/451087/articles/549337-monthlyrainfall-forecasting-by-a-hybrid-neural-network-of-discrete-wavelet-transform-and-deeplearning

\section{Hosted file}

Figures.pdf available at https://authorea.com/users/451087/articles/549337-monthly-rainfallforecasting-by-a-hybrid-neural-network-of-discrete-wavelet-transform-and-deep-learning

\section{Hosted file}

Tables.docx available at https://authorea.com/users/451087/articles/549337-monthly-rainfallforecasting-by-a-hybrid-neural-network-of-discrete-wavelet-transform-and-deep-learning 